\title{
Aplicación de la prueba t-Student para la competencia técnica y trazabilidad analítica: ejemplo de estudio
}

Application of the t-Student test for technical competition and analytical traceability: example of study

Recibido: junio 22 de 2018 | Revisado: julio 26 de 2018 | Aceptado: agosto 15 de 2018

\author{
George Argota Pérez ${ }^{\mathrm{I}}$ \\ Humberto Argota Coello ${ }^{2}$
}

1 Centro de Investigaciones Avanzadas y Formación Superior en Educación, Salud y Medio Ambiente "AMTAWI". Puno, Perú. george.argota@gmail.com

2 Laboratorio de Minerales. Empresa Geominera Oriente. Santiago de Cuba, Cuba. h.argota.coello@gmail.com

\begin{abstract}
Resumen
La toma decisiones en múltiples ocasiones requiere de un sustento estadístico comparativo. El propósito del estudio fue aplicar la prueba t-Student para la competencia técnica y trazabilidad analítica mediante un ejemplo de estudio. Dada una muestra de referencia certificada (DOLT-3: Dogfish Liver) se evaluó la competencia técnica de dos especialistas (A y B) mediante el reporte de sus datos que estuvieron basados en los estadígrafos: media, desviación estándar, coeficiente de variación, rango, sesgo estandarizado y la curtosis estandarizada. Asimismo, la trazabilidad de un método para el análisis del elemento $\mathrm{Cu}$ fue mediante el uso de la prueba t-Student. Independientemente de los hallazgos, lo pretendido fue mostrar cómo pudo confiarse en los resultados comparativos considerando el estadígrafo paramétrico, t-Student.
\end{abstract}

Palabras clave: prueba t-Student, competencia técnica, muestra de referencia certificada, ensayo acreditado

\begin{abstract}
Decision making requires on multiple occasions of a comparative statistical support. The purpose of the study was to apply the t-Student test for technical competence and analytical traceability using an example study. Given a certified reference sample (DOLT-3: Dogfish Liver) the technical competence of two specialists (A and B) was evaluated by reporting their data based on the following statisticians: mean, standard deviation, coefficient of variation, range, standardized bias and standardized kurtosis. Likewise, the traceability of a method for the analysis of the $\mathrm{Cu}$ element was achieved through the use of the t-Student test. Regardless of the findings, the aim was to show how the comparative results could be relied on considering the parametric statistic, t-Student.
\end{abstract}

Key words: t-Student test, technical competence, certified reference sample, accredited test 


\section{Introducción}

En la actualidad, el uso de pruebas estadísticas representa una herramienta fundamental para la toma de decisiones. La prueba t-Student es una de las más utilizadas para la comparación de dos muestras en diferentes escenarios de interés. Li et al., (2018), estudiaron la selección robusta de los grados de libertad con la distribución t-Student mediante la estimación adaptativa de modelo múltiple.

En otro estudio se analizó la predicción de la señal de enlace (en redes sociales) mediante factorización bayesiana probabilística de la matriz variable con la t-Student (Wang et al., 2017). Asimismo, Sauer et al., (2019) utilizaron la t-Student para realizar una comparación empírica entre el control remoto y las pruebas de campo clásicas. En el caso de Yago et al., (2018) se utilizaron la t-Student para el reconocimiento de la información sobre el proceso de aprendizaje de estudiantes en múltiples escenarios.

Por su parte, Ruggeri, Alimonti \& Bocca (2016), utilizaron la prueba t-Student para la completa validación y acreditación de un método y así respaldar, los estudios de biomonitoreo en humano para elementos traza y ultra trazas.

El propósito del estudio fue aplicar la prueba t-Student para la competencia técnica y trazabilidad analítica mediante un ejemplo de estudio.

\section{Materiales y métodos}

- Análisis de la muestra de referencia certificada
Se utilizó como muestra de referencia certificada (MRC) para metales: DOLT-3 (Dogfish Liver) y se analizó la concentración de $\mathrm{Cu}(31,2 \pm 1,00)$. Para el desempeño técnico debió seleccionarse uno de los especialistas (A y B) de un laboratorio X. A cada uno se les entregó la muestra donde realizaron 10 réplicas en diferentes momentos y finalmente, se les evaluó como estadígrafos los siguientes: media, desviación estándar, coeficiente de variación, rango, sesgo estandarizado y la curtosis estandarizada.

Para comparar las medias con relación a la MR se siguió la siguiente fórmula:

- $\mathrm{t}_{\mathrm{cal}}=\mathrm{X}_{\mathrm{CRM}}-\mathrm{X}_{\text {método }} / \sqrt{\left(\mathrm{U}_{\mathrm{CRM}} / \mathrm{k}\right)^{2}+\left(\mathrm{S}_{1} / \sqrt{\mathrm{n})^{2}}\right.}$

Donde:

a) Si $\mathrm{t}_{\mathrm{cal}}<\mathrm{t}_{(\mathrm{a}, \mathrm{g} . \mathrm{l})}$ (tabulado): entonces el método es trazable y puedes calcular su incertidumbre.

b) $\mathrm{X}_{\mathrm{CRM}}$ : valor medio de la muestra de referencia (comunicación en el certifico)

c) $\mathrm{X}_{\text {método }}$ : valor medio de las 10 réplicas realizadas a las muestras de referencia (empleando el método del ensayo acreditar)

d) $\mathrm{U}_{\mathrm{CRM}}$ : valor de incertidumbre de la muestra de referencia (comunicación en el certifico)

e) K: valor establecido (por lo general, es 2)

f) S: desviación estándar (10 valores) que se les realizó a la muestra de referencia empleando el método de ensayo acreditar 
g) N: número de réplica realizada a la muestra de referencia (según el ejemplo, es 10)

h) g,l: grado de libertad (según el ejemplo, es 9)
- Análisis de los datos

Se utilizó el programa estadístico Statgraphics Centurion versión 18. Mediante el menú comparación se seleccionó el análisis de dos muestras independientes (Figura 1).

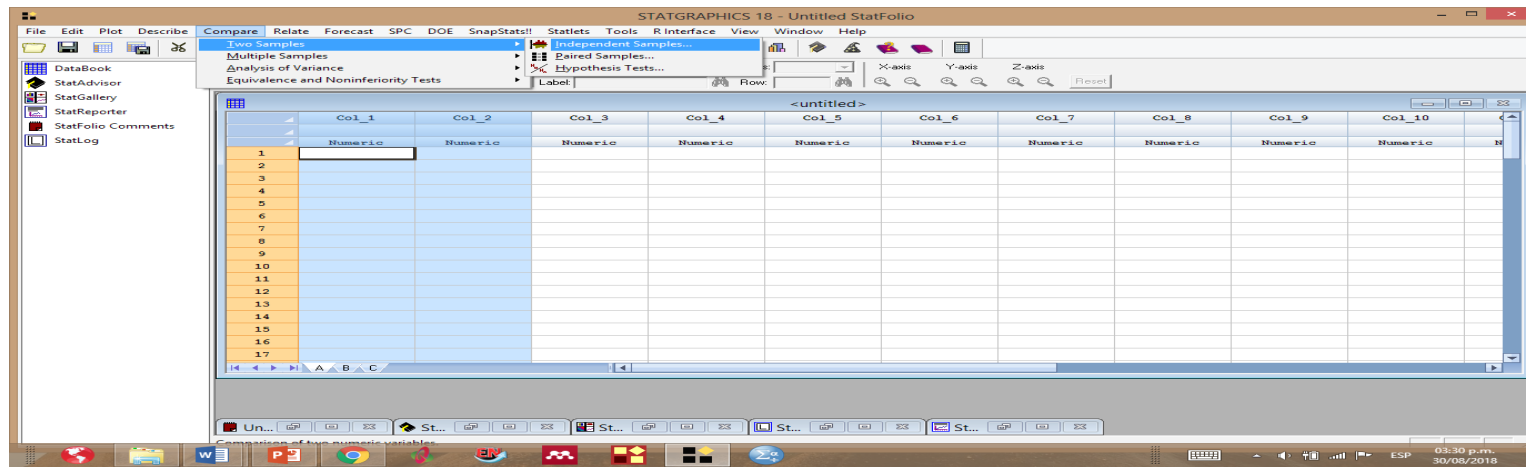

Figura 1. Comparación de dos muestras / Statgraphics Centurion versión 18

\section{Resultados y discusión}

La Tabla 1 muestra los resultados de la muestras de referencias obtenidos por tres especialistas químicos en el laboratorio X.

Tabla 1

Competencia profesional entre especialistas DOLT-3 $\mathrm{Cu}=31,2 \pm 1,00$

\begin{tabular}{ccc} 
& Especialista A & Especialista B \\
\hline & 31,60 & 31,40 \\
& 32,20 & 32,10 \\
& 30,80 & 30,20 \\
& 30,90 & 30,60 \\
& 30,01 & 29,80 \\
& 30,70 & 30,30 \\
& 31,10 & 31,30 \\
& 32,02 & 31,20 \\
& 30,00 & 29,70 \\
& 29,90 & 29,80 \\
\hline X & $\mathbf{3 0 , 2 9}$ & $\mathbf{3 0 , 6 4}$ \\
DS & $\mathbf{0 , 8 2}$ & $\mathbf{0 , 8 2}$ \\
CV & $\mathbf{2 , 6 8}$ & $\mathbf{2 , 6 7}$ \\
Rango & $\mathbf{2 , 4}$ & $\mathbf{2 , 3}$ \\
SE & $\mathbf{0 , 6 1}$ & $\mathbf{0 , 3 5}$ \\
CE & $\mathbf{- 0 , 6 3}$ & $\mathbf{- 0 , 7 2}$ \\
\hline
\end{tabular}

\section{Comparación de Medias}

- Intervalos de confianza del 95,0\% para la media del especialista A: 30,923 +/- 0,589958 (30,333; $31,513)$

- Intervalos de confianza del 95,0\% para la media del especialista A: 30,64 +/- 0,587195 [30,0528, 31,2272]

- Suponiendo varianzas iguales: 0,283 $+/-0,773047 \quad(-0,490047 ; 1,05605)$

Prueba t para comparar medias

- Suponiendo varianzas iguales: $\mathrm{t}=$ 0,769115 valor $\mathrm{P}=0,4518$

Puesto que el intervalo $(-0,490047$ hasta 1,05605) contiene el valor de 0 , no hay diferencia significativa entre las medias de los especialistas con un nivel de confianza del 95,0\%.

Aunque los especialistas mostraron similitud estadística en el reporte analítico con relación al valor promedio de la muestra de referencias (especialista A: suponiendo varianzas iguales: $\mathrm{t}=-1,05697$; 
valor $\mathrm{P}=0,304504$; especialista $\mathrm{B}$ : suponiendo varianzas iguales: $\mathrm{t}=-2,1468$ valor $\mathrm{P}=0,0456848$ ) se seleccionó al especialista $\mathrm{B}$, pues la medición de sus datos estuvieron más próximos (concentrados) al valor de la media muestral.

Por otra parte, al suponerse que el contenido de $\mathrm{Cu}$ en el hígado del pez es perjudicial, a partir de una concentración de 35,4+1,05 ug/Kg de peso húmedo, la trazabilidad del $\mathrm{Cu}$ puede verificarse utilizando el MRC $(31,2 \pm \mathbf{1 , 0 0} \mathbf{u g} / \mathrm{Kg})$. Asimismo, en el certifico de la MR se indicó que, la incertidumbre se calculó utilizando un factor de cobertura $\mathrm{K}=2$ y donde la MRC se analizó 10 veces en el laboratorio $\mathrm{X}$ en condiciones de precisión intermedia y siguiendo el protocolo del método analítico (diferentes series y variando los equipos como especialistas). En la Tabla 2 se muestra el resultado de cada mediación.

\section{Tabla 2}

Concentración analitica de Cu (ug/Kg de peso búmedo)

\begin{tabular}{cc}
\hline No. de análisis & Resultados \\
\hline 1 & 1,35 \\
2 & 1,34 \\
3 & 1,40 \\
4 & 1,36 \\
5 & 1,35 \\
6 & 1,33 \\
7 & 1,31 \\
8 & 1,34 \\
9 & 1,32 \\
10 & 1,34 \\
\hline $\mathbf{X}$ & $\mathbf{1 , 3 4 4}$ \\
$\mathbf{D S}$ & $\mathbf{0 , 0 2 4 6}$ \\
\hline
\end{tabular}

Al asumir todos los cálculos se obtuvo que,

$$
\text { - } \mathrm{t}_{\text {calculada }}=0,725
$$

El valor de $t_{\text {calculada }}$ al compararse con el $\mathrm{t}_{\text {tabulado }}$ de dos colas para $\mathrm{n}-1$ (9 g.l y alfa $=$ al $5 \%$ ) entonces, el valor de $t_{\text {tabulado }}$ de es 2,26 y por tanto, los resultados indicados por el método analítico pueden considerarse trazables al valor de la MRC debido a que, el $\mathrm{t}_{\text {tabulado }}>\mathrm{t}_{\text {calculada }}$.

Una vez que la trazabilidad fue verificada puede asumirse la misma con los resultados obtenidos para analizar muestras de hígado (análisis de rutina con similitud en términos de concentración y matriz analítica). Resulta fundamental que, el método analítico siga estando bajo condiciones de aseguramiento de la calidad para sí, certificar, a través del tiempo que los resultados continuarán siendo trazables.

Finalmente, el uso de la prueba t-Student, constituye una herramienta analítica para la toma decisiones no solo en la comparación de muestras ambientales, además, durante el control de cualquier procedimiento que se desee verificar. 


\section{Referencias}

Li, Q., Ben, Y., Tan, J., Mohsen N. S. \& Chambers, J. (2018). Robust selection of the degrees of freedom in the Student's $t$ distribution through Multiple Model Adaptive Estimation. Signal Processing; 153, 255-265. https://doi. org/10.1016/j.sigpro.2018.07.023

Ruggieri, F., Alimonti, A. \& Bocca, B. (2016). Full validation and accreditation of a method to support human biomonitoring studies for trace and ultra-trace elements. Trends in Analytical Chemistry; 80, 471485. http://dx.doi.org/10.1016/j. trac.2016.03.023

Sauer, J., Sonderegger, A., Heyden, K., Biller, J., Klotz, J. \& Uebelbache, A. (2019). Extra-laboratorial usability tests: An empirical compar- ison of remote and classical field testing with lab testing. Applied Ergonomics; 74, 85-96. https://doi. org/10.1016/j.apergo.2018.08.011

Wang, Y., Liu, F., Tao, X.S. \& Wu, J. (2017). Link sign prediction by Variational Bayesian Probabilistic Matrix Factorization with Student-t Prior. Information Sciences; 405, 175-189. http://dx.doi. org/10.1016/j.ins.2017.04.014

Yago, H., Clemente, J., Rodriguez, D., Fernandez de Cordoba, P. (2018). ON-SMMILE: Ontology Network-based Student Model for Multiple Learning Environments. Data \& Knowledge Engineering; 1-37. https://doi.org/10.1016/j. datak.2018.02.002 